\title{
A sphingosine-1-phosphate receptor 1 agonist inhibits tertiary lymphoid tissue reactivation and hypersensitivity in the lung
}

\author{
CA Huppé ${ }^{1}$, P Blais Lecours ${ }^{1}$, A Lechasseur ${ }^{1}$, DR Gendron ${ }^{1}$, AM Lemay ${ }^{1}$, EY Bissonnette ${ }^{1,2}$, \\ MR Blanchet ${ }^{1,2}$, C Duchaine ${ }^{1,3}$, MC Morissette M $^{1,2}$, Rosen $^{4}$ and D Marsolais ${ }^{1,2}$
}

Hypersensitivity pneumonitis is characterized by pulmonary accumulation of B-cell-rich tertiary lymphoid tissues (TLTS), which are alleged sites of amplification for antigen-specific responses. The sphingosine-1-phosphate receptor $1\left(\mathrm{~S}_{1} \mathrm{P}_{1}\right)$ regulates key mechanisms underlying lymphoid tissue biology and its chemical modulation causes lymphocyte retention in lymph nodes. Given the putative immunopathogenic impact of lymphocyte accumulation in TLTs, we investigated whether or not chemical modulation of $\mathrm{S}_{1} \mathrm{P}_{1}$ caused lymphocyte retention within TLTs in a model of hypersensitivity pneumonitis. Mice were exposed subchronically to Methanosphaera stadtmanae (MSS) in order to induce an hypersensitivity pneumonitis-like disease. MSS exposure induced B-cell-rich TLTs surrounded by

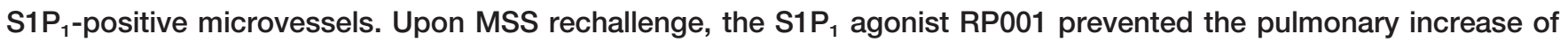
CXCL13, a chief regulator of B-cell recruitment in lymphoid tissues. This was associated with a complete inhibition of MSS rechallenge-induced TLT enlargement and with a 2.3-fold reduction of MSS-specific antibody titers in the lung. Interference with TLT reactivation was associated with a $77 \%$ reduction of neutrophil accumulation and with full inhibition of protein-rich leakage in the airways. Thus, an $\mathrm{S}_{1} \mathrm{P}_{1}$ agonist hinders TLT enlargement upon antigenic rechallenge and inhibits key pathognomonic features of experimental hypersensitivity pneumonitis.

\section{INTRODUCTION}

Chronic exposure to bioaerosols containing a heavy antigenic burden can lead to respiratory diseases such as hypersensitivity pneumonitis (HP). ${ }^{1,2} \mathrm{HP}$ is a chronic inflammatory condition characterized by both humoral and cellular components. Repeated exposures to causative agents induce lymphocytic infiltrates, local release of antigen-specific antibodies, and an ensuing accumulation of granulocytes. ${ }^{3,4}$ Upon antigenic reencounter leading to an HP exacerbation, diffuse alveolar damage caused by granulocytic inflammation and protein-rich exudate is reminiscent of dyspnea, which can be mild to fatal., So far, the only effective treatment for HP is to avoid eliciting antigens. ${ }^{7}$ Yet, antigen avoidance is often difficult to implement for social and economical reasons; and sometimes impossible when causative agents cannot be identified. ${ }^{2}$ Thus, there is a strong interest in identifying regulatory points with potential for alleviation and reversal of pulmonary hypersensitivity as it is seen in HP.

Mononucleated cell accumulation foci, often referred to as tertiary lymphoid tissues (TLTs), are routinely found adjacent to the vascular or bronchial stroma in pulmonary hypersensitivity diseases, including HP. ${ }^{8,9}$ Pulmonary TLTs are generally composed of a follicle-like B-cell center often associated with a T-cell zone, ${ }^{10}$ although their structure can vary according to the causative agent and the state of TLT maturation. ${ }^{11}$ Accordingly, it is currently believed that the mechanisms of TLT formation in the lung depend on the nature of the causative agent. ${ }^{10}$ Still, these different mechanisms often converge toward the pulmonary release of lymphangiogenic factors and homeostatic chemokines including tumor necrosis factor (TNF) receptor

${ }^{1}$ Centre de recherche de I'Institut Universitaire de cardiologie et de pneumologie de Québec, Université Laval, Quebec City, Quebec, Canada. ${ }^{2}$ Faculty of Medecine Department of Medecine, Université Laval, Quebec City, Quebec, Canada. ${ }^{3}$ Faculty of Science and Engineering, Department of Biochemistry, Microbiology, and Bioinformatics, Université Laval, Quebec City, Quebec, Canada and ${ }^{4}$ Department of Chemical Physiology, The Scripps Research Institute, La Jolla, California, USA. Correspondence: D Marsolais, david.marsolais@criucpq.ulaval.ca 
ligands and chemokines with potent T-cell- and B-cellattracting properties. ${ }^{11-17}$ In $\mathrm{HP}$, the current theory suggests that TLTs promote B-cell-mediated immunity in the lung and thus favor antigen-triggered HP exacerbations. ${ }^{10}$ Therefore, targeting pulmonary TLTs could impact on the progression of HP.

Sphingosine-1-phosphate (S1P) is a bioactive lipid regulating key inflammatory mechanisms through activation of five high-affinity $\mathrm{G}$-protein coupled receptors $\left(\mathrm{S}_{1} \mathrm{P}_{1}-\mathrm{S}_{1} \mathrm{P}_{5}\right)$. Given that pharmacological activation of $\mathrm{S}_{1} \mathrm{P}_{1}$ induces lymphocyte sequestration in secondary lymphoid organs, ${ }^{18,19}$ we initially hypothesized that an $\mathrm{S}_{1} \mathrm{P}_{1}$ agonist would also promote the enlargement of pulmonary TLTs. According to the current theory, ${ }^{10}$ lymphocyte retention in TLTs would be associated with maintenance of the antigen-specific response in the lung and eventually, with the enhancement of HP signs. Alternatively, $\mathrm{S}_{1} \mathrm{P}_{1}$-mediated interference with the release of cytokines in the lung ${ }^{20,21}$ might alleviate TLT reactivation and subsequently blunt the hypersensitivity response.

The aim of the study was to determine the role of $\mathrm{S}_{1} \mathrm{P}_{1}$ in the reactivation of TLTs in response to Methanosphaera stadtmanae (MSS), a recently described immunogenic agent found at high concentration in agricultural environments. ${ }^{22}$ We show that, contrary to its effect on lymph nodes, an $\mathrm{S}_{1} \mathrm{P}_{1}$ agonist profoundly reduces the enlargement of TLTs in response to a MSS rechallenge and alleviates the cardinal features of experimental HP.

\section{RESULTS}

MSS exposure induces partially organized B-cell-rich TLTs Following subchronic exposure to MSS, peribronchial and perivascular mononuclear cellular infiltrates are induced in the lung. ${ }^{22}$ We found that these cellular infiltrates are compatible with the type II TLT morphology described by Fleige et al. ${ }^{11}$ with a high prevalence of B220-positive B cells and dispersed T cells, and/or T-cell aggregates (Figure 1a). In agreement with previous literature, ${ }^{23,24}$ we also observed the presence of CD11c-positive macrophages/dendritic cells within TLTs (Figure 1b). PNAd-positive vessels were detected in the periphery of TLTs, which is compatible with the occurrence of high endothelial venules (Figure 1c). Lyve-1 staining revealed no lymphatic endothelial cells in the core of the TLTs. Instead, Lyve-1-positive cells were broadly distributed among the pulmonary parenchyma (Figure 1d). These observations confirm the notion that subchronic exposure to MSS induces the formation of early stage, yet spatially organized, TLTs.

\section{An S1P $_{1}$ agonist inhibits TLT amplification upon MSS recall} Given that $\mathrm{S}_{1} \mathrm{P}_{1}$ agonists induce lymphocyte sequestration in lymph nodes, ${ }^{18,25}$ we tested the hypothesis that activation of $\mathrm{S}_{1} \mathrm{P}_{1}$ would prevent lymphocyte exit from TLTs, thus causing TLT enlargement. Control mice that remained unexposed to MSS did not present any pulmonary TLT (not shown). On day 22 (3 days after micropump implantation), TLTs were found throughout the lung in association with blood vessels and/or
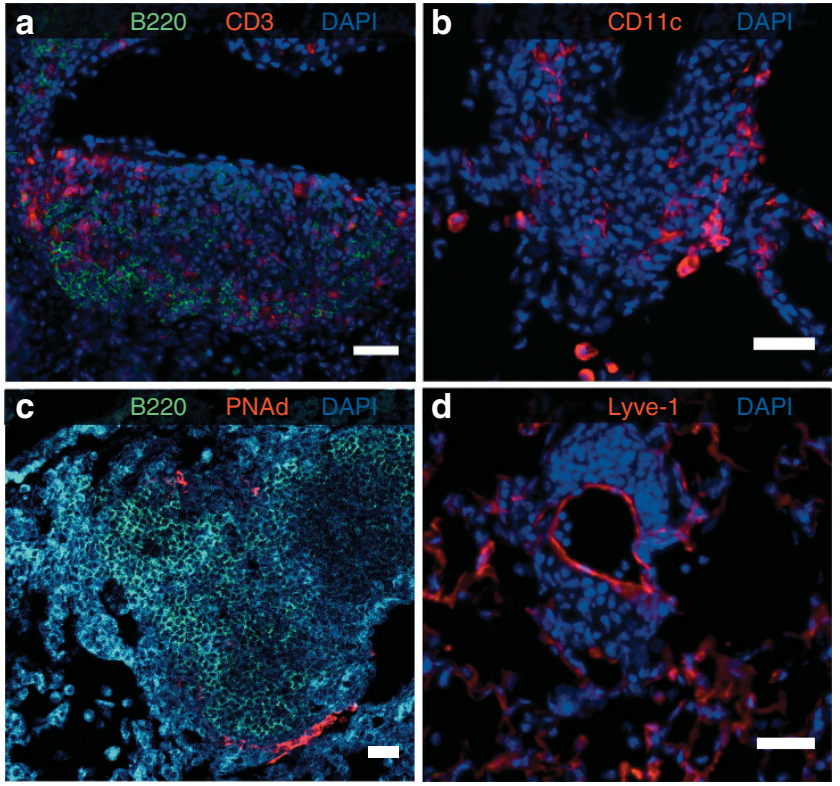

Figure 1 Subchronic exposure to MSS induces TLT formation. Mice were sensitized for 3 weeks to MSS and euthanized 4 days following the last MSS exposure. Rounded nuclei (blue) dense area compatible with TLTs were mainly composed of (a) B220 ${ }^{+} \mathrm{B}$ cells (green) and $\mathrm{CD} 3^{+} \mathrm{T}$ cells (red); (b) featured occasional $\mathrm{CD} 11 \mathrm{c}^{+}$macrophages/dendritic cells (red); and (c) displayed peripheral $\mathrm{PNAd}^{+}$high endothelial venule-like structures. (d) The Lyve $-1^{+}$lymphatic endothelial cell marker was only found on capillary-like structures outside of rounded nuclei dense areas. Fluorescence level was adjusted based on primary antibody omission controls. Images are representative of at least three independent lung sections from three different mice in two independent experiments. Scale bars: $20 \mu \mathrm{m}$. MSS, Methanosphaera stadtmanae; TLT, tertiary lymphoid tissues.

bronchi (Figure $\mathbf{2 b}$ i, ii). At that time, the area occupied by TLTs was not modulated by RP001, when compared to the vehicle (Figure 3), in spite of systemic lymphopenia (Supplementary Figure 1 online). Between day 22 and day 24 , there was a nearly $50 \%$ decrease in the area occupied by TLTs, independently of the administration of RP001 (Figure 2b iii, iv; Figure 3). MSS rechallenge induced a nearly three-fold increase of TLT area on day 24, and this increase was completely inhibited by RP001 (Figure 2b v, vi; Figure 3).

In view of the profound inhibition of MSS-induced reactivation of TLTs by the $S 1 P_{1}$ agonist, we investigated which cell subsets were affected (Figure 4). In agreement with immunofluorescence results (Figure 1a), the predominant lymphocyte subset found in the pulmonary parenchyma of mice exposed to MSS was B cells, followed by CD4 ${ }^{+} \mathrm{T}$ cells and $\mathrm{CD}^{+} \mathrm{T}$ cells. When compared to the mock-rechallenged group, the parenchyma of MSS-rechallenged mice featured a 1.6-fold increase in average B-cell numbers $\left(6.8 \pm 1.1 \times 10^{5}\right.$ vs. $1.1 \pm 0.1 \times 10^{6}$ cells, respectively); a 1.9 -fold increase in CD ${ }^{+}$ T-cell numbers $\left(2.4 \pm 0.4 \times 10^{5}\right.$ vs. $4.5 \pm 0.3 \times 10^{5}$ cells, respectively); and a two-fold increase in $\mathrm{CD}^{+}$T-cell numbers $\left(0.9 \pm 0.2 \times 10^{5}\right.$ vs. $1.7 \pm 0.2 \times 10^{5}$ cells, respectively). RP001 reduced the numbers of these three lymphocyte subsets to the level of mock-rechallenged mice (Figure 4b-d). In opposition to its inhibitory effect on antigen- 
a

\begin{tabular}{|c|c|c|c|c|c|c|c|c|c|c|}
\hline & T & $T$ & $T$ & $T$ & $T$ & $T$ & $T$ & $T$ & † & 7 \\
\hline Days & 12 & 3 & 8 & 10 & 15 & 16 & 17 & 19 & 22 & 24 \\
\hline & \llcorner & & & $\gamma$ & & & $\downarrow$ & 1 & Rechallenge & Euthanasia \\
\hline
\end{tabular}

b
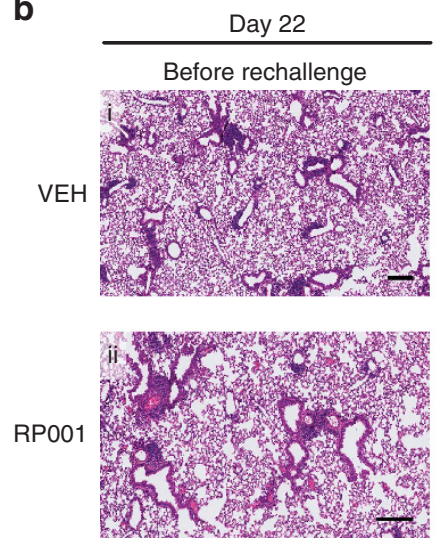

Day 24
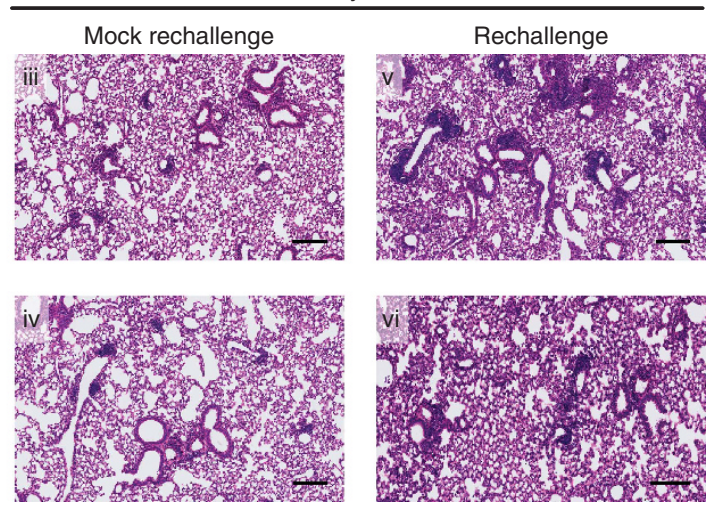

Figure 2 Histological modifications induced by MSS and RP001. (a) Mice were instilled i.n. with saline or saline with $2 \mathrm{mg} \mathrm{ml}^{-1}$ of MSS for 3 weeks as described in methods. On day 19, mice were treated with the vehicle or the short-acting S1 $\mathrm{P}_{1}$ agonist, RP001 (1 mg kg ${ }^{-1}$ per day) for 5 days using osmotic subcutaneous micropumps. On day 22, mice were either euthanized (i, ii), mock-rechallenged (iii, iv) or rechallenged with MSS ( $v$, vi) and euthanized on day 24. (b) Hematoxylin and eosin-stained sections representative of five independent mice per experimental group from two independent experiments are shown. Scale bars: $200 \mu$ m. i.n., intranasally; MSS, Methanosphaera stadtmanae.

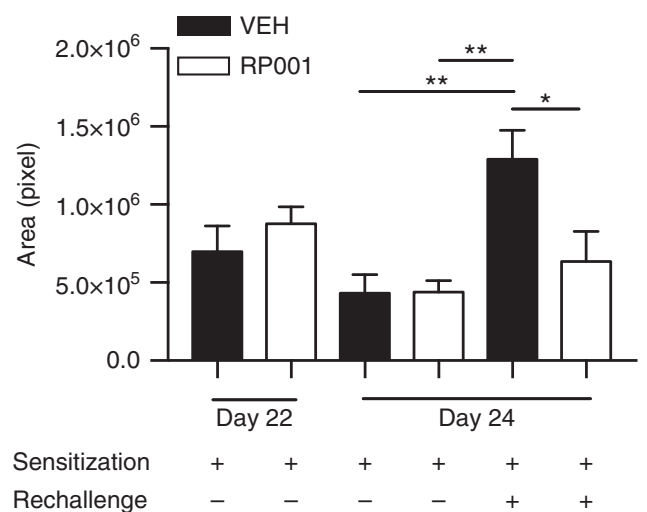

Figure $3 \quad \mathrm{~S}_{1} \mathrm{P}_{1}$ agonism prevents TLT reactivation upon MSS rechallenge. Quantification of MSS-induced TLTs in the lung of MSSsensitized mice treated with the vehicle (black bars) or RP001 (white bars) and euthanized on day 22 or on day 24 following mock ( - ) or MSS $(+)$ rechallenge. Images were digitalized and the number of pixels occupied by individual TLTs was quantified and summed up for each animal in order to obtain an index of TLT areas. Results are expressed as means \pm s.e.m. Three mice per group on day 22 and five mice per group on day 24 . Results were replicated in two independent experiments. $P$-value: ${ }^{*}<0.05$, ${ }^{* *}<0.01$. MSS, Methanosphaera stadtmanae; TLT, tertiary lymphoid tissues.

triggered TLT expansion, RP001 induced a nearly two-fold increase in the total cell number of peripheral (inguinal) lymph nodes in mice sensitized and rechallenged with MSS (Figure 5a). This effect was mainly attributed to lymph node retention of $B$ cells (Figure $\mathbf{5 b}$ and $\mathbf{c}$ ).

Importantly, we found that $\mathrm{S}_{1} \mathrm{P}_{1}$ distribution in TLTs differed from that of lymph nodes. For instance, $\mathrm{S}_{1} \mathrm{P}_{1}$-eGFP is present on lymphocytes and reticular structures within lymph nodes (Figure 6a). However, we found poor $\mathrm{S}_{1} \mathrm{P}_{1}-\mathrm{eGFP}$ signal within TLTs (Figure 6b and Supplementary Movie 1) as well as few $\mathrm{S}_{1} \mathrm{P}_{1}$-positive lymphocytes within these structures (not shown). Instead, $\mathrm{S}_{1} \mathrm{P}_{1}$ is distributed in the periphery of TLTs, on structures resembling the capillary network and co-localizing with endothelial cell marker CD31 (Figure 6c). In addition, we observed CD19-positive cells in conjunction with $\mathrm{S}_{1} \mathrm{P}_{1}$-positive capillary-like structures in the periphery of TLTs (Figure 6d). Thus, $\mathrm{S} \mathrm{P}_{1}$ is broadly expressed among the lung parenchyma and although it is found in the periphery of TLTs, its penetration within these structures is limited.

\section{RP001 inhibits MSS rechallenge-induced accumulation of CXCL13 in the lung}

Specific soluble factors influence the impact of S1P pathway modulators in different types of lymphoid organs, ${ }^{26,27}$ as well as TLT formation and maintenance. ${ }^{11,12,14,15,17}$ We thus initially screened for modulation of messenger RNA levels encoding for CXCL12, CXCL13, TNFS13B (BAFF), TNFS14 (LIGHT), and TNF in response to MSS (Supplementary Figure 2), which are central regulators of B-cell accumulation and TLT formation. ${ }^{11,12,14,15,17}$ Among these candidates, CXCL13 displayed the highest messenger RNA level increase in response to MSS. Moreover, the messenger RNA level for CXCL13 was significantly decreased by RP001 in mice rechallenged with MSS (Supplementary Figure 3). In agreement with these results, neither the MSS rechallenge nor the $\mathrm{S}_{1} \mathrm{P}_{1}$ agonist significantly affected the concentration of TNF (Figure 7a) or CXCL12 (Figure 7b) in MSS-sensitized mice. However, RP001 tended to increase the concentration of TNF and CXCL12 in the mock-rechallenged group, when compared to the vehicle (Figure 7b). In the vehicle-treated group, MSS rechallenge nearly doubled the accumulation of CXCL13 (12.4 \pm $\left.0.8 \mathrm{pg} \mathrm{mg}^{-1}\right)$, when compared to mock rechallenge (6.1 \pm 


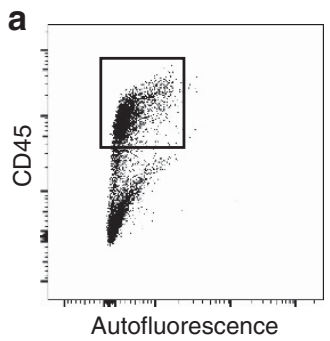

b

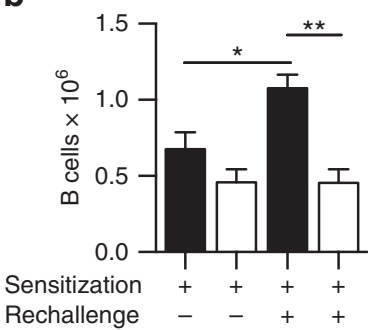

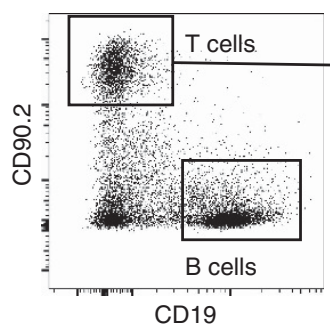

C

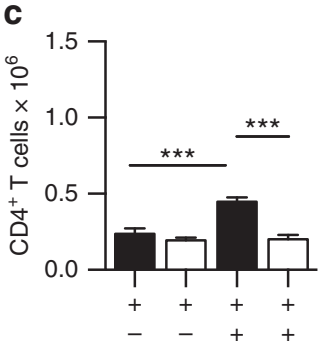

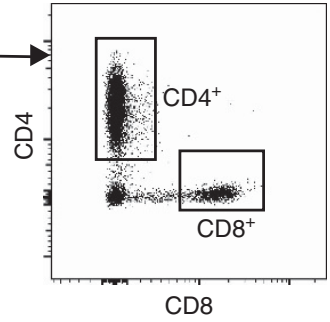

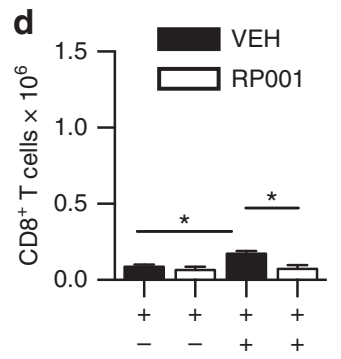

Figure 4 The $\mathrm{S} 1 \mathrm{P}_{1}$ agonist RP001 inhibits lymphocyte accumulation in the lung. The accumulation of lymphocyte subsets was assessed in the pulmonary parenchyma of mice treated with the vehicle (black bars) or with RP001 (white bars) and euthanized on day 24 , i.e., $48 \mathrm{~h}$ following mock ( - ) or MSS $\left(+\right.$ ) rechallenge. (a) Gating strategy. B cells were identified as CD45 ${ }^{+} \mathrm{CD} 90.2^{-} \mathrm{CD} 19^{+}$cells. T cells were gated from the CD45 ${ }^{+} \mathrm{CD} 90.2^{+} \mathrm{CD} 19^{-}$ population and separated based on the differential expression of CD4 and CD8. Frequencies of cell subsets obtained by flow cytometry were multiplied by the total number of cells isolated from digested lungs to compute the absolute number of (b) B cells; (c) CD4 ${ }^{+} \mathrm{T}_{\text {cells, and; (d) CD8 }}{ }^{+}$T cells. Results are expressed as means \pm s.e.m. $n=5$ mice per group. Results were replicated in two independent experiments. $P$-value: ${ }^{*}<0.05,{ }^{* *}<0.01,{ }^{* \star *}<0.001$. MSS, Methanosphaera stadtmanae.
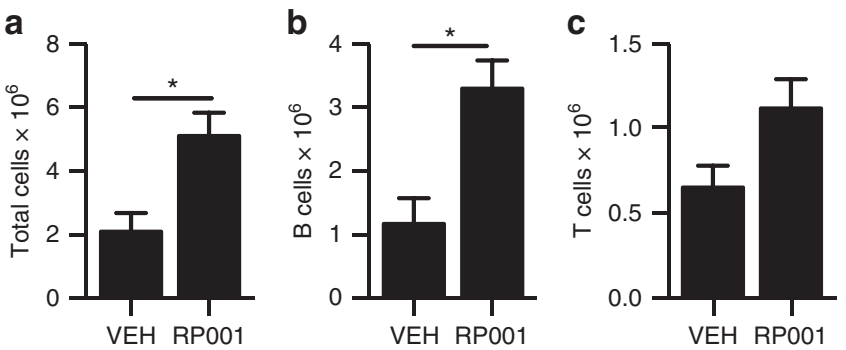

Figure 5 RP001 increases lymphocyte numbers in inguinal lymph nodes. The accumulation of leukocyte subsets on day 24 was assessed in inguinal lymph nodes of MSS-sensitized and rechallenged mice treated with the VEH or RP001 as described in the Methods section. Frequencies of cell subsets obtained by flow cytometry were multiplied by (a) total cell numbers in digested inguinal lymph node to calculate absolute number of (b) B cells based on the frequency of the $\mathrm{CD} 45^{+} \mathrm{CD} 90.2^{-} \mathrm{CD} 19^{+}$population and (c) T cells based on the frequency of the CD45 ${ }^{+}$CD $90.2^{+}$ CD19- population. Results are expressed as means \pm s.e.m. $n=5$ mice per group. $P$-value: * $<0.05$. MSS, Methanosphaera stadtmanae; VEH, vehicle.

$0.7 \mathrm{pg} \mathrm{mg}^{-1}$ ), and this increase was inhibited by RP001 $\left(7.9 \pm 0.9 \mathrm{pg} \mathrm{mg}^{-1}\right)$. Similar to CXCL12 and TNF, RP001 per se mildly increased CXCL13 concentration in the lung of mock-rechallenged mice, when compared to vehicle $(P=0.054)$ (Figure 7c). Yet, upon MSS rechallenge, RP001 fully inhibited the increase of CXCL13, supporting the instrumental role for this chemokine in the inhibition of TLT reactivation by RP001.

\section{RP001 alleviates critical features of MSS-induced HP-like experimental disease}

B lymphocytes-derived antigen-specific antibodies drive the inflammatory reaction in HP. ${ }^{7}$ We addressed the concept that inhibition of B-cell accumulation upon MSS rechallenge by RP001 alleviates the local antigen-specific response. We found that, on day 24, MSS-specific antibody titers were reduced by nearly 2.3-fold by RP001 (Figure 8a and b), when compared to the group receiving MSS rechallenge and the vehicle treatment. We then investigated if reduction of the antigen-specific antibody titers led to an alleviation of key pathognomonic features of HP.

As expected, the bronchoalveolar lavage fluid (BALF) from control mice (receiving mock sensitization and mock rechallenge) contained low average cell numbers mainly composed of macrophages $\left(1.8 \pm 0.3 \times 10^{4}\right.$ cells $)$, and RP001 did not significantly modify BALF cellular content under these conditions (Figure 8c). Mice sensitized with MSS for 3 weeks and receiving a mock rechallenge displayed increased macrophage numbers in BALF, whether they were treated with vehicle or RP001 $\left(2.3 \pm 0.4 \times 10^{5}\right.$ and $2.4 \pm 0.6 \times 10^{5}$ cells, respectively). MSS sensitization also increased BALF lymphocytes in mice treated with vehicle or RP001 $\left(6.6 \pm 1.7 \times 10^{4}\right.$ and $9.0 \pm 0.9 \times 10^{4}$ cells, respectively) (Figure $8 \mathrm{c}$ ), while no significant accumulation of neutrophils was detected. However, MSS rechallenge strongly increased neutrophil accumulation in $\mathrm{BALF}$, reaching an average number of $1.6 \pm 0.3 \times 10^{5}$ cells. RP001 treatment led to a potent $77 \%$ inhibition of MSSinduced neutrophil accumulation, which was not different from the level found in mock-rechallenged mice (Figure 8c). This was accompanied by a full reversal of MSS rechallengeinduced albumin accumulation in BALF (Figure 8d). RP001 thus potently inhibits the hypersensitivity response induced by MSS along with determining features of diffuse alveolar damage. 

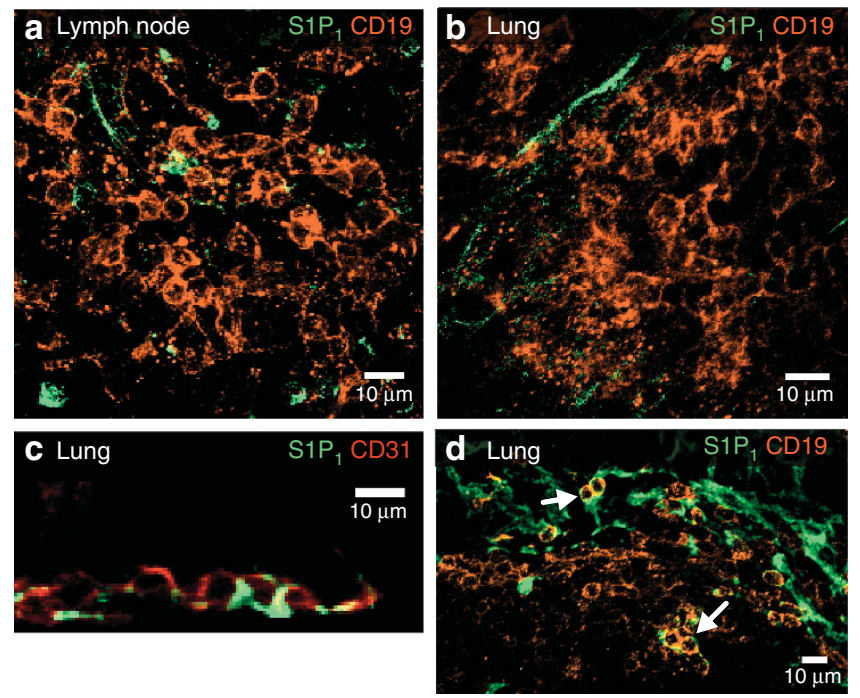

Figure $6 \mathrm{~S} 1 \mathrm{P}_{1}$ distribution in TLTs and lymph nodes. Wild-type and transgenic $\mathrm{S1P}_{1}$-eGFP mice were sensitized to MSS for 3 weeks. Four days after the last exposure, anti-CD19 or anti-CD31 antibodies were intravenously injected and mice were euthanized $30 \mathrm{~min}$ later. (a) B cells (orange) and $\mathrm{S}^{\mathrm{P}_{1}}$ (green) distribution in the mediastinal lymph node. (b) The B-cell- (orange) rich TLT shows low penetration of $\mathrm{S}_{1} \mathrm{P}_{1}$ (green) (see also Supplementary Movie 1). (c) $\mathrm{S}_{1} \mathrm{P}_{1}$ signal co-localizes with vessellike structures positive for endothelial cell marker CD31 (red) in the lung. (d) B cells (orange) are located within capillary-like vessels (white arrow) expressing $\mathrm{S}_{1} \mathrm{P}_{1}$ (green) in the periphery of the TLTs. Fluorescence level was adjusted based on wild-type or primary antibody omission controls. Shown images of anti-CD19 in the lung are representative of at least five independent mice from five independent experiments; anti-CD31 and lymph node images are representative of at least three independent mice from three independent experiments. MSS, Methanosphaera stadtmanae; $\mathrm{S}_{1} \mathrm{P}_{1}$, sphingosine-1-phosphate receptor $1 ; \mathrm{TLT}$, tertiary lymphoid tissues.

\section{DISCUSSION}

TLTs are commonly found in chronic diseases. ${ }^{8,9,28-30}$ The body of literature on the subject reveals an unsuspected complexity of the mechanisms governing their genesis as well as their involution. ${ }^{11-13,16,23,24,31,32}$ In fact, the anatomical location ${ }^{10,17,33}$ and triggering agents ${ }^{11}$ appear to define the underlying mechanisms of TLT regulation supporting the diversity, the specificity, and the likely differing functional impacts of these lymphoid structures. Our study unravels a new player in TLT biology. We found that $\mathrm{S}_{1} \mathrm{P}_{1}$ chemical modulation does not promote TLT enlargement under basal conditions, but prevented TLT reactivation upon an antigenic rechallenge. Moreover, our findings support the concept that TLTs are a source of local factors that promote antigen-induced hypersensitivity in the lung. This study provides the first evidence for S1P pathway regulation of TLTs and defines S1P as a sufficient target to alleviate critical features underlying diffuse alveolar damage as it is seen in the context of HP.

Pulmonary TLTs possess an overall lower degree of organization, when compared to secondary lymphoid tissues. ${ }^{10}$ In agreement with this notion, we determined that in the lung, $\mathrm{S} \mathrm{P}_{1}$ is mainly found on the capillary network as well as on structures resembling blood vessels located outside of the TLTs. Moreover, we found limited penetration of $\mathrm{S}_{1} \mathrm{P}_{1}$ within the TLT
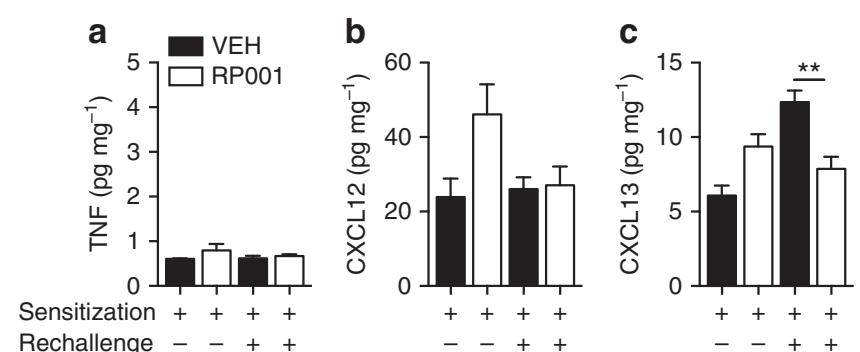

Figure 7 RP001 inhibits MSS rechallenge-induced CXCL13 increase in the lung. Enzyme-linked immunosorbent assays were performed on lung homogenates from tissue isolated on day 24 from MSS-sensitized and mock or MSS-rechallenged mice treated with the vehicle (VEH; black bars) or RP001 (white bars). Levels of TNF (a), CXCL12 (b), and (c) CXCL13 are expressed relatively to the weight of lung tissue. Results are expressed as means \pm s.e.m. $n=5$ mice per group. Results were replicated in two independent experiments. $P$-value: ${ }^{\star *}<0.01$. MSS, Methanosphaera stadtmanae; TNF, tumor necrosis factor.

itself. This contrasts with the prior observation that $\mathrm{S}_{1} \mathrm{P}_{1}$ is found on organized lymphatic and vascular stromal cells located within lymph nodes. ${ }^{25}$ Another striking difference between lymph nodes and TLTs is that TLTs do not feature a defined capsule that restricts cellular entry and exit at very limited locations, i.e., in blood or lymphatic vessels. ${ }^{28,34}$ Moreover, highly compartmentalized S1P pools in these organized lymphatic vessels guide lymphocyte exit from lymph nodes. ${ }^{35}$ This mechanism of lymphocyte exit is thus unlikely to be operational in TLTs given their lower degree of organization. This is further supported by the lack of effect of RP001 on TLT size under basal conditions, given that $\mathrm{S}_{1} \mathrm{P}_{1}$ chemical modulators induce lymphocyte sequestration by disrupting $\mathrm{S}_{1} \mathrm{P}_{1}$-mediated guidance of lymphocytes toward the S1P-rich lymph found in lymphatic vessels. ${ }^{36}$ Together, our current results thus argue against the concept that lymphocytes exit pulmonary TLTs using $\mathrm{S}_{1} \mathrm{P}_{1}$-guided migration toward compartmentalized S1P into lymphatic vessels. It is important to note that the TLT featured in our model may not be fully mature and that the inhibitory response to $S 1 \mathrm{P}_{1}$ modulators may depend on the state of TLT maturation.

Although TLT maintenance is unaffected by $\mathrm{S}_{1} \mathrm{P}_{1}$ chemical modulation in the current system, antigen-driven TLT enlargement is nevertheless inhibited by RP001. One mechanism to explain this effect is the induction of lymphopenia and sequestration of lymphocytes in distant lymphoid organs. ${ }^{19,37}$ However, sphingosine analog-induced lymphopenia was shown to mainly impact on naive lymphocytes ${ }^{38}$ and to marginally influence total lymphocyte numbers in lymphoid organs insensitive to sphingosine analog-induced sequestration. ${ }^{37}$ On the other hand, profound inhibition of pulmonary accumulation of lymphocytes by $\mathrm{S}_{1} \mathrm{P}_{1}$ agonists was associated with the inhibition of local release of potent chemotactic agents. ${ }^{21}$

Similarly, TLT enlargement in response to immunostimulating factors can be mediated by a number of secreted proteins including $\mathrm{T}$-cell- and B-cell-stimulating factors including LIGHT $^{17}$ and BAFF; ${ }^{15}$ and homeostatic chemokines including CXCL12 $2^{11}$ and CXCL13. ${ }^{12,14,31}$ Among these likely candidates, 
a

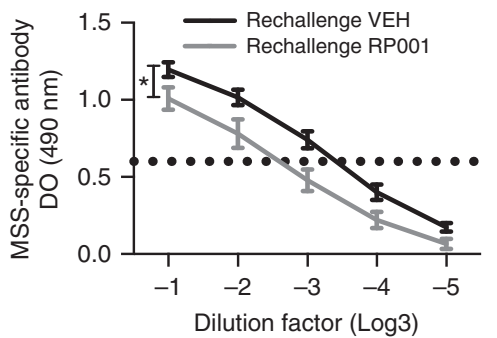

b

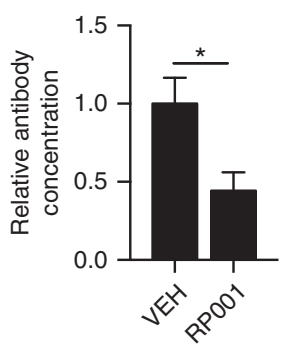

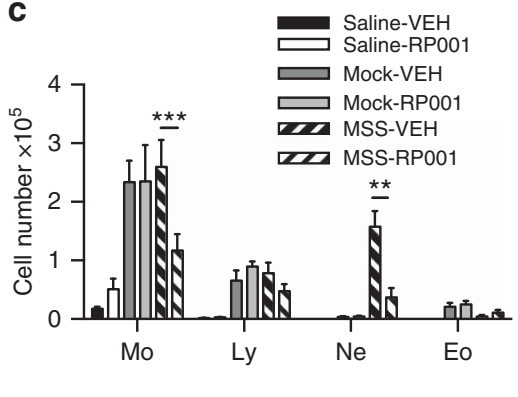

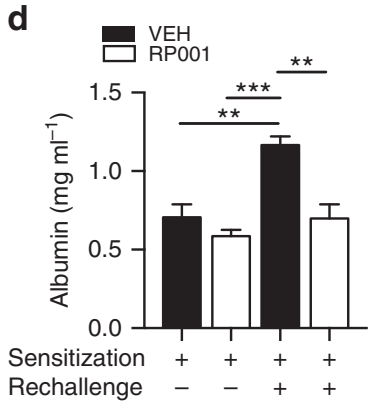

Figure 8 RP001 prevents the reactivation of MSS-induced hypersensitivity and inhibits determinants of diffuse alveolar damage. Mice were sensitized to MSS and treated with the VEH or with RP001 as described in the Methods section. Mice were euthanized on day 24 , i.e., $48 \mathrm{~h}$ following mock or MSS rechallenge. (a) MSS-specific lgG/lgM/lgAs were quantified by indirect enzyme-linked immunosorbent assay in supernatants of lung homogenates. (b) Relative antibody concentration was determined at the optical density of 0.6. (c) Quantification of leukocyte subsets in BALF including Mo, Ly, Ne, and Eo. (d) Albumin was quantified in BALF as a surrogate for plasma-rich leakage into the airways. Results are expressed as means \pm s.e.m. $n=5$ mice per group, except for saline groups in which $n=3$ mice per group. Results were replicated in two independent experiments except for albumin quantification that was performed in one experiment. $P$-value: ${ }^{*}<0.05,{ }^{* *}<0.01,{ }^{* * *}<0.001$. BALF, bronchoalveolar lavage fluid; Eo, eosinophils; Ig, immunoglobulin; Ly, lymphocytes; Mo, macrophages; MSS, Methanosphaera stadtmanae; Ne, neutrophils; VEH, vehicle.

we found the potent B-cell chemotactic agent CXCL13 to be the most significantly increased in response to MSS, and this was detected at both the messenger RNA and the protein level. Of course, the possibility remains that mediators not addressed in the current study could also contribute to the RP001-mediated inhibition of TLT reactivation. Yet, our observation that RP001 inhibits CXCL13 accumulation in response to the MSS rechallenge, in combination to the prior observation that CXCL13 neutralization is sufficient to alter TLT formation supports an instrumental role for CXCL13 in the RP001mediated inhibition of TLT expansion. Moreover, our findings are consistent with the view that disruption of specific chemokine-chemokine receptor axes by S1P receptor modulators can influence lymphocyte trafficking in lymphoid tissues. $^{26,27}$ Given the high S1P ${ }_{1}$-eGFP signal on endothelial cells and the role of these cells in the regulation of pulmonary inflammation, ${ }^{20}$ it is tempting to speculate that the inhibition of TLT reactivation by RP001 involves $\mathrm{S}_{1} \mathrm{P}_{1}$-mediated inhibition of CXCL13 expression by endothelial cells, but this theory remains to be tested.

This is the first evidence supporting the concept that the S1P pathway could be utilized to counteract an HP-like disease. Still, the use of additional and more conventional HP-triggering agents is required in order to clarify the pre-clinical potential of $\mathrm{S}_{1} \mathrm{P}_{1}$ chemical modulation for this class of diseases, which encompass a diversity of causative agents. ${ }^{7}$ Of note, $\mathrm{S}_{1} \mathrm{P}_{1}$ agonists were shown to interfere with a number of diseases using specific mechanisms of action. For instance, $\mathrm{S}_{\mathrm{P}} \mathrm{P}_{1}$ alleviation of experimental autoimmune encephalomyelitis likely involves induction of lymphopenia as well as $\mathrm{S}_{1} \mathrm{P}_{1}$ degradation in the central nervous system; ${ }^{39,40} \mathrm{~S} \mathrm{P}_{1}$ prevention of endothelial cell-derived cytokine release alleviates influenza virus-induced immunopathological damages; ${ }^{20}$ and $\mathrm{S}_{1} \mathrm{P}_{1^{-}}$ mediated inhibition of chemokines specific to activated $\mathrm{T}$ cells are associated with inhibition of $\mathrm{TH} 2$ eosinophilic inflammation in experimental asthma. ${ }^{21}$ In the case of HPlike diseases, the immunopathological response specifically results from a prolonged exposure to a high antigenic load. This leads to a TH1-TH17 response combined to the release of antigen-specific antibodies that promote local neutrophilic inflammation through formation of immune complexes, and activation of local Fc-receptor-bearing cells. ${ }^{2,7}$ Most importantly, TLTs are a major source of antibodies, ${ }^{41}$ but their ability to generate a local antigen-specific response in the context of $\mathrm{HP}$ remained unclear. In this study, we show that an $\mathrm{S}_{1} \mathrm{P}_{1}$ agonist reduces TLT reactivation and B-cell numbers in the lung in response to MSS, leading to a decreased local amount of antigen-specific antibodies. Although our results do not rule out the possibility that immunoregulatory agents acting as dampeners of inflammation could derive from TLTs, they are consistent with the notion that TLTs are a local source of antigen-specific immunoglobulins involved in the amplification of hypersensitivity-derived neutrophilic inflammation.

In agreement with the sufficient role of $\mathrm{S}_{1} \mathrm{P}_{1}$ in the stabilization of the endothelial barrier, ${ }^{42,43}$ our findings also support the concept that an $\mathrm{S}_{1} \mathrm{P}_{1}$ agonist could interfere with antigenic rechallenge-induced fluid exudation into the alveoli, which is another determining mechanism of the diffuse alveolar damage that is associated with dyspnea. Thus in the context of $\mathrm{HP}, \mathrm{S}_{\mathrm{P}} \mathrm{P}_{1}$ agonists would provide beneficial effects by interfering with B-cell accumulation in TLTs and by limiting fluid exudation in the airways.

Our results do not support that RP001 promotes the enlargement of moderately organized pulmonary TLTs. However, we found that upon an antigenic rechallenge, RP001 efficiently interferes with TLT reactivation and reduces antigen-specific antibody titers in the lung. This is associated with potent inhibition of neutrophilic inflammation and protein-rich leakage into the airways, which are two major determinants of diffuse alveolar damage. Thus, an $\mathrm{S}_{1} \mathrm{P}_{1}$ agonist interferes with TLT reactivation and alleviates critical pathognomonic features of an experimental HP-like disease.

\section{METHODS}

Preparation of M. stadtmanae. M. stadtmanae cultures (Strain \#3091, DMSZ, Braunschweig, Germany) were expanded under anaerobic 
conditions in Methanosphaera medium (MCB-3) containing ruminal fluid (graciously provided by Centre de recherche en science animale de Deschambault, Quebec, Canada). Following MSS expansion, cells were washed, lyophilized, stored at $4{ }^{\circ} \mathrm{C}$ until reconstitution in sterile saline solution and sonicated to obtain a single-cell suspension as described. ${ }^{22}$ Since MSS is an obligatory anaerobic organism, there are no living cells in the solution instilled to mice, similarly to what is found in the air of contaminated environments.

Animals. Eight weeks old C57Bl/6 female mice were obtained from Jackson laboratories (Bar Harbor, ME) and kept in an environment free of specific pathogens (Centre de recherche de l'Institut Universitaire de cardiologie et de pneumologie de Québec, Quebec City, Quebec, Canada). Experimental protocols, care, and handling procedures were reviewed and approved by the Animal Care Committee of Université Laval (approval \# 2014084) under the authority of the Canadian Council on Animal Care.

Airway exposure protocol. Mice were anesthetized with isoflurane and instilled intranasally once daily, 3 consecutive days per week, for 3 weeks with saline or with a saline solution containing $2 \mathrm{mg} \mathrm{ml}^{-1}$ of MSS. ${ }^{22}$ Two days after the last MSS exposure, osmotic micropumps (Durect, Cupertino, CA, USA) containing the vehicle or the shortacting $\mathrm{S}_{1} \mathrm{P}_{1}$ agonist RP001 ( $1 \mathrm{mg} \mathrm{kg}^{-1}$ per day; Tocris, Avounmouth, Bristol, UK) were implanted subcutaneously under sterile condition. Three days later, mice were either euthanized with ketamine/xylazine overdose or re-exposed to vehicle (mock procedure) or to MSS (rechallenged group), and euthanized $48 \mathrm{~h}$ later.

Bronchoalveolar lavage fluid analyses. After euthanasia, right lobe was ligated, trachea was cannulated with an 18-gauge needle and the left lobe was washed three times with $0.5 \mathrm{ml}$ of phosphate-buffered saline. Total cell numbers in BALF were quantified using a hemocytometer and differential counts were performed on cytospins after cells staining with the Hemacolor kit (Thermofisher, Waltham, MA, USA).

Histology. The left lobe was fixed for $72 \mathrm{~h}$ in $4 \%$ paraformaldehyde and embedded in paraffin. Paraffin-embedded coronal lung sections were stained with hematoxylin and eosin as described. ${ }^{44}$ Whole-lung slices were digitalized and analyzed using the Image J software $(\mathrm{NIH}$, Bethesda, MD, USA) in order to quantify the total area occupied by TLTs.

Immunofluorescence. Wild-type mice were sensitized to MSS as described above and euthanized 4 days following the last MSS exposure. After euthanasia, the left lobes were removed and fixed in phosphate-buffered saline containing $4 \%$ paraformaldehyde and $10 \%$ sucrose for $2-4 \mathrm{~h}$ at $4{ }^{\circ} \mathrm{C}$. Following fixation, lungs were transferred into a $30 \%$ sucrose solution for $72 \mathrm{~h}$. Lungs were then embedded in optimum cutting temperature compound, frozen in liquid nitrogen and stored at $-80^{\circ} \mathrm{C}$ until $5 \mu \mathrm{m}$-thick sections were overlaid on glass slides using a cryostat. Tissues were then incubated in phosphatebuffered saline containing $1 \%$ bovine serum albumin (BSA), $2 \%$ goat serum, and $0.05 \%$ Tween-20 for $45 \mathrm{~min}$. Slides were labeled with combinations of the following antibodies: anti-B220 FITC (RA3-6B2; BD Pharmingen, San Jose, CA), biotinylated anti-PNAd (MECA-79; Biolegend, San Diego, CA), biotinylated anti-CD3 (1452-C11; BD Pharmingen), anti-CD11c APC (HL3; BD Pharmingen), rat anti-Lyve1 IgG (ALY7; eBioscience, San Diego, CA), goat anti-rat IgG-AF555 (Life Technologies, Carlsbad, CA), and streptavidin-Cy3 (Biolegend). According to Kocks et al., ${ }^{13}$ TLTs were identified on immunofluorescence preparations as areas composed of dense rounded nuclei, consistent with lymphocyte morphology, found in the vicinity of a blood vessel or a bronchus.

In order to visualize $\mathrm{S}_{1} \mathrm{P}_{1}$ and TLTs in the lung, $\mathrm{S}_{\mathrm{P}} \mathrm{P}_{1}$-eGFP knock-in mice ${ }^{25}$ were exposed to MSS as indicated above. Mice were injected intravenously with $0.5 \mathrm{mg} \mathrm{kg}^{-1}$ of PE-coupled anti-CD19 antibody (6D5; Biolegend) or APC-coupled anti-CD31 antibody (MEC13.3;
Biolegend) $30 \mathrm{~min}$ prior to euthanasia. Mediastinal lymph nodes and the left pulmonary lobes were removed. Lobes were cut in $2 \mathrm{~mm}$-thick slices and both lymph nodes and lung slices were processed to whole mounts and visualized with a confocal microscope (LSM800, Zeiss, Oberkochen, Germany).

Flow cytometry. Lungs were perfused free of blood, minced and digested with $0.1 \%$ collagenase IV, $0.2 \%$ trypsin inhibitor, and $0.02 \%$ DNase for $90 \mathrm{~min}$ at $37^{\circ} \mathrm{C}$. Tissues were triturated every $15 \mathrm{~min}$ in order to obtain a single-cell suspension. Red blood cells were lysed and single-cell suspensions were passed through a $70 \mu \mathrm{m}$ strainer. Lymphocyte composition was analyzed by flow cytometry, after an enrichment procedure performed with a 30\% Percoll gradient (GE Healthcare, Uppsala, Sweden), using primary antibodies raised against CD45 (30-F11; Biolegend), CD19 (1D3; Abcam, Cambridge, UK), CD90.2 (30-H12; Biolegend), CD4 (RM4-5; Biolegend), and CD8 (53-6.7; Biolegend). Data were acquired using a FACS Diva-driven LSR Fortessa (BD Biosciences, Franklin Lake, NJ). Results were analyzed with the FlowJo software (Tree Star, Ashland, OR, USA). Absolute numbers of cell subsets were computed by multiplying the number of cells obtained using an hemocytometer (crystal violet staining) by the frequencies of cell subsets obtained from flow cytometric analyses.

Immunoglobulin titration. MSS-specific antibody level was measured using indirect enzyme-linked immunosorbent assay on supernatants from lung homogenates. Plates coated with $50 \mu \mathrm{g}$ MSS per ml were blocked with $2 \%$ BSA and incubated for $2 \mathrm{~h}$ with serial dilutions of lung homogenates. After incubation, plates were washed three times and overlaid with a biotinylated goat anti-mouse immunoglobulin $\mathrm{M}$ (IgM), IgA, and IgG (ab102460; Abcam) for $2 \mathrm{~h}$ and then washed three times. Plates were incubated with a streptavidin-coupled horseradish peroxidase for $20 \mathrm{~min}$ and revealed using OPD (Sigmafast, SigmaAldrich, St Louis, MO, USA).

ELISA. Snap-frozen lungs were homogenized by sonication (Sonifier cell disruptor 185; Branson, Danbury, CT) in phosphate-buffered saline containing protease inhibitors (complete EDTA-free protease inhibitor Cocktail, Roche, Mississauga, ON, Canada). After centrifugation, supernatants were stored at $-80^{\circ} \mathrm{C}$. Quantification of CXCL12 (R\&D system, Minneapolis, MN), TNF (Biolegend), and CXCL13 (R\&D system) was performed according to the instructions of the manufacturer.

Statistical analyses. Data were expressed using means \pm s.e.m. When required, a log transformation was used to homogenize the variance between experimental groups. Unpaired bi-directional $t$-test was used when appropriate; and one-way analysis of variances were used for multiple comparisons. The Bonferroni procedure was employed for post hoc analyses. Statistical significance was set at $P \leqslant 0.05$.

SUPPLEMENTARY MATERIAL is linked to the online version of the paper at http://www.nature.com/mi

\section{ACKNOWLEDGMENTS}

This work was supported by NSERC grant 402151 and funding from the Respiratory Health Network of the FRQ-S. C-AH and DRG received scholarships from the Training program of the Respiratory Health Network of the FRQ-S- IRSC and from the FRQ-S. E.B., M.-R.B., C.D., M.C.M. and D.M. are members of the FRQ-S Respiratory Health Network and D.M., M.-R.B. and C.D. received FRQ-S research scholar awards. We would like to thank Marc Veillette at the IUCPQ Research Center flow cytometry core facility for his expertise and technical assistance. We also thank Émilie Bernatchez for her contribution to the culture of archaeal species. This study was supported by the NSERC Grant 402151.

\section{AUTHOR CONTRIBUTIONS}

C.-A.H., P.B.L., M.C.M., C.D., H.R. and D.M.: conception and design; C.-A.H., P.B.L., A.L., D.R.G. and A.-M.L.: acquisition of data; C.-A.H., 
P.B.L., A.L., M.C.M., H.R. and D.M.: analysis and interpretation of data; C.-A.H., P.B.L., M.C.M. and D.M.: drafting the manuscript for important intellectual content; C.-A.H., P.B.L., A.L., D.R.G., A.-M.L., E.B., M.-R.B., C.D., M.C.M., H.R. and D.M.: substantial involvement in its revision.

\section{DISCLOSURE}

Hugh Rosen is a consultant to Celgene. The remaining authors declare no conflict of interest.

c 2018 Society for Mucosal Immunology

\section{REFERENCES}

1. Prevention CoDCa. Work-Related Lung Disease, Surveillance Report, 2007. National Institute for Occupational Safety and Health (2007); https:// www.cdc.gov/niosh/docs/2008-143/; accessed on April 7th 2017.

2. Tarlo, S., Cullinan, P. \& Nemery, B. Occupational and Environmental Lung Diseases: Diseases from Work, Home, Outdoor and Other Exposures (Wiley-Blackwell, Oxford, 2010).

3. Girard, M., Israël-Assayag, E. \& Cormier, Y. Pathogenesis of hypersensitivity pneumonitis. Curr. Opin. Allergy Clin. Immunol. 4, 93-98 (2004).

4. Spagnolo, P. et al. Hypersensitivity pneumonitis: a comprehensive review. J. Investig. Allergol. Clin. Immunol. 25, 237-250 (2015). quiz follow 50.

5. Miyazaki, Y., Tateishi, T., Akashi, T., Ohtani, Y., Inase, N. \& Yoshizawa, Y. Clinical predictors and histologic appearance of acute exacerbations in chronic hypersensitivity pneumonitis. Chest 134, 1265-1270 (2008).

6. Tasaka, S. et al. Fatal diffuse alveolar damage from bird fanciers' lung. Respiration 64, 307-309 (1997).

7. Lacasse, Y., Girard, M. \& Cormier, Y. Recent advances in hypersensitivity pneumonitis. Chest 142, 208-217 (2012).

8. Suda, T. et al. Development of bronchus-associated lymphoid tissue in chronic hypersensitivity pneumonitis. Chest 115, 357-363 (1999).

9. Rangel-Moreno, J., Hartson, L., Navarro, C., Gaxiola, M., Selman, M. \& Randall, T.D. Inducible bronchus-associated lymphoid tissue (BALT) in patients with pulmonary complications of rheumatoid arthritis. J. Clin. Invest. 116, 3183-3194 (2006).

10. Randall, T.D. Bronchus-associated lymphoid tissue (BALT) structure and function. Adv. Immunol. 107, 187-241 (2010).

11. Fleige, $\mathrm{H}$. et al. IL-17-induced $\mathrm{CXCL} 12$ recruits $B$ cells and induces follicle formation in BALT in the absence of differentiated FDCs. J. Exp. Med. 211, 643-651 (2014).

12. Rangel-Moreno, J. et al. The development of inducible bronchusassociated lymphoid tissue depends on IL-17. Nat. Immunol. 12, 639-646 (2011).

13. Kocks, J.R., Davalos-Misslitz, A.C., Hintzen, G., Ohl, L. \& Förster, R. Regulatory T cells interfere with the development of bronchus-associated lymphoid tissue. J. Exp. Med. 204, 723-734 (2007).

14. Bracke, K.R. et al. Role of CXCL13 in cigarette smoke-induced lymphoid follicle formation and chronic obstructive pulmonary disease. Am. J. Respir. Crit. Care Med. 188, 343-355 (2013).

15. Seys, L.J. et al. Role of B cell-activating factor in chronic obstructive pulmonary disease. Am. J. Respir. Crit. Care Med. 192, 706-718 (2015).

16. Demoor, T. et al. Role of lymphotoxin-alpha in cigarette smoke-induced inflammation and lymphoid neogenesis. Eur. Respir. J. 34, 405-416 (2009).

17. Lee, $Y$. et al. Recruitment and activation of naive $T$ cells in the islets by lymphotoxin beta receptor-dependent tertiary lymphoid structure. Immunity 25, 499-509 (2006).

18. Mandala, S. et al. Alteration of lymphocyte trafficking by sphingosine-1phosphate receptor agonists. Science 296, 346-349 (2002).

19. Sanna, M.G. et al. Sphingosine 1-phosphate (S1P) receptor subtypes S1P1 and S1P3, respectively, regulate lymphocyte recirculation and heart rate. J. Biol. Chem. 279, 13839-13848 (2004).

20. Teijaro, J.R. et al. Endothelial cells are central orchestrators of cytokine amplification during influenza virus infection. Cell 146, 980-991 (2011).

21. Marsolais, D., Yagi, S., Kago, T., Leaf, N. \& Rosen, H. Modulation of chemokines and allergic airway inflammation by selective local sphingosine1-phosphate receptor 1 agonism in lungs. Mol. Pharmacol. 79, 61-68 (2011).
22. Blais Lecours, P. et al. Immunogenic properties of archaeal species found in bioaerosols. PLoS ONE 6, e23326 (2011).

23. Halle, S. et al. Induced bronchus-associated lymphoid tissue serves as a general priming site for T cells and is maintained by dendritic cells. J. Exp. Med. 206, 2593-2601 (2009).

24. GeurtsvanKessel, C.H. et al. Dendritic cells are crucial for maintenance of tertiary lymphoid structures in the lung of influenza virus-infected mice. J. Exp. Med. 206, 2339-2349 (2009).

25. Cahalan, S.M. et al. Actions of a picomolar short-acting S1P1 agonist in S1P1-eGFP knock-in mice. Nat. Chem. Biol. 7, 254-256 (2011).

26. Yopp, A.C. et al. FTY720-enhanced Tcell homing is dependent on CCR2, CCR5, CCR7, and CXCR4: evidence for distinct chemokine compartments. J. Immunol. 173, 855-865 (2004).

27. Yopp, A.C., Ochando, J.C., Mao, M., Ledgerwood, L., Ding, Y. \& Bromberg, J.S. Sphingosine 1-phosphate receptors regulate chemokinedriven transendothelial migration of lymph node but not splenic T cells. J. Immunol. 175, 2913-2924 (2005).

28. Hwang, J.Y., Randall, T.D. \& Silva-Sanchez, A. Inducible bronchusassociated lymphoid tissue: taming inflammation in the lung. Front. Immunol. 7, 258 (2016).

29. Hogg, J.C. et al. The nature of small-airway obstruction in chronic obstructive pulmonary disease. N. Engl. J. Med. 350, 2645-2653 (2004).

30. Perros, F. et al. Pulmonary lymphoid neogenesis in idiopathic pulmonary arterial hypertension. Am. J. Respir. Crit. Care Med. 185, 311-321 (2012).

31. Rangel-Moreno, J., Moyron-Quiroz, J.E., Hartson, L., Kusser, K. \& Randall, T.D. Pulmonary expression of CXC chemokine ligand 13, CC chemokine ligand 19, and CC chemokine ligand 21 is essential for local immunity to influenza. Proc. Natl. Acad. Sci. USA 104, 10577-10582 (2007).

32. Moyron-Quiroz, J.E. et al. Persistence and responsiveness of immunologic memory in the absence of secondary lymphoid organs. Immunity 25 , 643-654 (2006).

33. Neyt, K., Perros, F., GeurtsvanKessel, C.H., Hammad, H. \& Lambrecht, B.N. Tertiary lymphoid organs in infection and autoimmunity. Trends Immunol. 33, 297-305 (2012).

34. Girard, J.P., Moussion, C. \& Förster, R. HEVs, lymphatics and homeostatic immune cell trafficking in lymph nodes. Nat. Rev. Immunol. 12, 762-773 (2012).

35. Schwab, S.R. \& Cyster, J.G. Finding a way out: Iymphocyte egress from lymphoid organs. Nat. Immunol. 8, 1295-1301 (2007).

36. Pappu, R. et al. Promotion of lymphocyte egress into blood and lymph by distinct sources of sphingosine-1-phosphate. Science 316, 295-298 (2007).

37. Luo, Z.J., Tanaka, T., Kimura, F. \& Miyasaka, M. Analysis of the mode of action of a novel immunosuppressant FTY720 in mice. Immunopharmacology 41, 199-207 (1999).

38. Hofmann, M., Brinkmann, V. \& Zerwes, H.G. FTY720 preferentially depletes naive $T$ cells from peripheral and lymphoid organs. Int. Immunopharmacol. 6, 1902-1910 (2006).

39. Garris, C.S. et al. Defective sphingosine 1-phosphate receptor 1 (S1P1) phosphorylation exacerbates TH17-mediated autoimmune neuroinflammation. Nat. Immunol. 14, 1166-1172 (2013).

40. Gonzalez-Cabrera, P.J. et al. S1P(1) receptor modulation with cyclical recovery from lymphopenia ameliorates mouse model of multiple sclerosis. Mol. Pharmacol. 81, 166-174 (2012).

41. Morissette, M.C. et al. Persistence of pulmonary tertiary lymphoid tissues and anti-nuclear antibodies following cessation of cigarette smoke exposure. Respir. Res. 15, 49 (2014).

42. Sanna, M.G. et al. Enhancement of capillary leakage and restoration of lymphocyte egress by a chiral S1P1 antagonist in vivo. Nat. Chem. Biol. 2, 434-441 (2006).

43. Marsolais, D. \& Rosen, H. Chemical modulators of sphingosine-1phosphate receptors as barrier-oriented therapeutic molecules. Nat. Rev. Drug Discov. 8, 297-307 (2009).

44. Fischer, A.H., Jacobson, K.A., Rose, J. \& Zeller, R. Hematoxylin and eosin staining of tissue and cell sections. CSH Protoc. 2008; doi:10.1101/ pdb.prot4986 (2008). 